\title{
A New Formalism for Representation of Binary Thermodynamic Data
}

\author{
J. P. HAJRA
}

The applicability of a formalism involving an exponential function of composition $x_{1}$ in interpreting the thermodynamic properties of alloys has been studied. The excess integral and partial molar free energies of mixing are expressed as:

$$
\begin{aligned}
& \Delta F^{x s}=a_{o} x_{1}\left(1-x_{1}\right) e^{b x_{1}} \\
& R T \ln \gamma_{1}=a_{o}\left(1-x_{1}\right)^{2}\left(1+b x_{1}\right) e^{b x_{1}} \\
& R T \ln \gamma_{2}=a_{o} x_{1}^{2}\left(1-b+b x_{1}\right) e^{b x_{1}}
\end{aligned}
$$

The equations are used in interpreting experimental data for several relatively weakly interacting binary systems. For the purpose of comparison, activity coefficients obtained by the subreg ular model and Krupkowski's formalism have also been computed. The present equations may be considered to be convenient in describing the thermodynamic behavior of metallic solutions.

VARIOUS analytical equations have been proposed to express activity coefficients as functions of composition for liquid alloys. Since extrapolations of activity coefficients with respect to composition are sometimes met with difficulties because of lack of enough data, the equations are of importance in analyzing the thermodynamic behavior of metallic solutions.

\section{THEORETICAL CONSIDERATIONS AND FORMALISMS}

Binary metallic solutions exhibiting regular behavior ${ }^{1}$ show symmetrical excess free energies of mixing with respect to composition. The regular solution model gives a reasonable description of a number of liquid metal systems where the two components are chemically similar in nature.

Many metallic solutions however, show deviations from the symmetric behavior. The subregular solution model $^{2}$ describes satisfactorily the situation where the partials at infinite dilution are not equal. The asymmetric behavior is introduced in the form of linear composition dependence of the interaction parameter. The equation is represented as

$$
\Delta F^{x s}=x_{1}\left(1-x_{1}\right)\left(a_{1}+a_{2} x_{1}\right)
$$

where $a_{1}=R T \ln \gamma_{1}^{o} ; a_{2}=R T\left(\ln \gamma_{2}^{o}-\ln \gamma_{1}^{o}\right)$ and $x_{1} \mathrm{~mol}$ fraction of component 1 .

The other equation involving two parameters has been proposed by Krupkowski. ${ }^{3}$ He has employed the following equations for activity coefficients for components 1 and 2 :

$$
\ln \gamma_{1}=w(T)\left(1-x_{1}\right)^{m}
$$

J. P. HAJRA is Lecturer, Department of Metallurgy, Indian Institute of Science, Bangalore - 560012 , India.

Manuscript submitted April 30, 1979.

$$
\begin{aligned}
\ln \gamma_{2}= & w(T)\left[\left(1-x_{1}\right)^{m}-\frac{m}{m-1}\left(1-x_{1}\right)^{m-1}\right. \\
& \left.+\frac{1}{m-1}\right]
\end{aligned}
$$

and hence $\Delta F^{x s}$ is represented as:

$$
\Delta F^{x s}=\frac{w(T)}{1-m}\left(1-x_{1}\right)\left[\left(1-x_{1}\right)^{m-1}-1\right]
$$

Asymmetry is due to a parameter $m$ in Eq. [4]. Darken $^{4}$ has shown that for certain binary systems a plot of $\log \gamma_{1}$ vs $\left(1-x_{1}\right)^{2}$ leads to three distinct regions: two terminal regions in which a linear relation exists, and an intermediate region which is difficult to represent by any simple polynominal relation.

\section{THE PRESENT EQUATION}

The present study investigates the form of the function $f(x)$ in Eq. [5], responsible for the asymmetric behavior of the excess integral free energies of mixing for various binary solutions:

$$
\frac{\Delta F^{x s}}{x_{1}\left(1-x_{1}\right)}=a_{o} f(x)
$$

The latter function is frequently expressed in the form of a polynomial consisting of compositional terms and corresponding coefficients which are important in describing the thermodynamic behavior of solutions. The polynomial in the present investigation is considered to be an exponential function of composition $x_{1}$. Hence $\Delta F^{x s}$ may be expressed as:

$$
\Delta F^{x s}=a_{o} x_{1}\left(1-x_{1}\right) e^{b x_{1}}
$$

where the constants $a_{o}$ and $b$ are to be evaluated for a particular system under consideration. The activity coefficients for components 1 and 2 are expressed by: 